\title{
Hybrid Metric-Palatini Gravity: Regular Stringlike Configurations
}

\author{
Kirill Bronnikov $1,2,3, * \mathbb{C}$, Sergey Bolokhov ${ }^{2}$ and Milena Skvortsova ${ }^{2}$ \\ 1 Center of Gravitation and Fundamental Metrology, VNIIMS, Ozyornaya ul. 46, Moscow 119361, Russia \\ 2 Institute of Gravitation and Cosmology, Peoples' Friendship University of Russia (RUDN University), \\ ul. Miklukho-Maklaya 6, Moscow 117198, Russia; boloh@rambler.ru (S.B.); milenas577@mail.ru (M.S.) \\ 3 Elementary Particle Physics Department, National Research Nuclear University "MEPhI", Kashirskoe sh. 31, \\ Moscow 115409, Russia \\ * Correspondence: kb20@yandex.ru
}

Received: 12 September 2020; Accepted: 9 October 2020; Published: 11 October 2020

check for updates

\begin{abstract}
We discuss static, cylindrically symmetric vacuum solutions of hybrid metric-Palatini gravity (HMPG), a recently proposed theory that has been shown to successfully pass the local observational tests and produce a certain progress in cosmology. We use HMPG in its well-known scalar-tensor representation. The latter coincides with general relativity containing, as a source of gravity, a conformally coupled scalar field $\phi$ and a self-interaction potential $V(\phi)$. The $\phi$ field can be canonical or phantom, and, accordingly, the theory splits into canonical and phantom sectors. We seek solitonic (stringlike) vacuum solutions of HMPG, that is, completely regular solutions with Minkowski metric far from the symmetry axis, with a possible angular deficit. A transition of the theory to the Einstein conformal frame is used as a tool, and many of the results apply to the general Bergmann-Wagoner-Nordtvedt class of scalar-tensor theories as well as $f(R)$ theories of gravity. One of these results is a one-to-one correspondence between stringlike solutions in the Einstein and Jordan frames if the conformal factor that connects them is everywhere regular. An algorithm for the construction of stringlike solutions in HMPG and scalar-tensor theories is suggested, and some examples of such solutions are obtained and discussed.
\end{abstract}

Keywords: hybrid metric-Palatini gravity; scalar-tensor theories; canonical and phantom scalar fields; cylindrically symmetric solutions; solitonic solutions

\section{Introduction}

General relativity (GR) is well known to be quite successful in describing local observational effects in the Solar system, in stellar astrophysics and, quite probably, in black hole physics. However, it faces serious problems at larger scales: on the galactic scale, it does not give a satisfactory explanation of the rotation curves without introducing the so-called Dark Matter (DM) of still unknown nature, and it cannot account for the observed accelerated expansion of the Universe without introducing the so-called Dark Energy (DE), which is an unknown kind of matter with large negative pressure. According to the latest observations, the energy content of the Universe consists, in terms of GR, of only about $5 \%$ of visible matter, about $20 \%$ of DM, and the remaining $75 \%$ of DE, see, e.g., [1,2].

In addition to attempts to solve the DM and DE problems in the framework of GR by introducing so far unobservable forms of DM, like WIMPs (weakly interacting massive particles) and various forms of DE like cosmological constant or "quintessence" scalar fields, etc., [3], there is an alternative broad trend, addressing the same problems while using various extensions of GR, such as, for instance, $f(R)$ theories, theories with additional scalar, vector and spinor fields, multidimensional theories, 
and those with extensions of the Riemannian geometry (metric-affine theories, those with Finslerian geometry, etc. [4,5]).

One of such recently proposed extensions of GR is the Hybrid Metric-Palatini Gravity (HMPG) [6]. In this theory, one assumes the independent existence of the Riemannian metric $g_{\mu v}$ and the connection $\hat{\Gamma}_{\mu \nu}^{\alpha}$. The total action of HMPG reads [6]

$$
S=\frac{1}{2 \varkappa^{2}} \int d^{4} x \sqrt{-g}[R+F(\mathcal{R})]+S_{m}
$$

where $R=R[g]$ is the Ricci scalar derived as usual from $g_{\mu v}$, while $F(\mathcal{R})$ is an arbitrary function of the scalar $\mathcal{R}=g^{\mu v} \mathcal{R}_{\mu v}$ that was obtained with the Ricci tensor $\mathcal{R}_{\mu \nu}$ calculated in the standard way from the connection $\hat{\Gamma}_{\mu v}^{\alpha}$; furthermore, $g=\operatorname{det}\left(g_{\mu v}\right), \varkappa^{2}$ is the gravitational constant, and $S_{m}$ is the action of all nongravitational matter.

HMPG, which combines the metric and Palatini approaches to the description of gravity and extends the formulation of $f(R)$ theories, has a number of achievements described in the reviews [7-9]. In particular, it agrees with the classical tests in the Solar system [10], fairly well solves the DM problem concerning the dynamics of galaxies and galaxy clusters; it has been shown to be able to describe an accelerating Universe without invoking a cosmological constant [11]. It is of interest that HMPG can be obtained from Palatini $R^{2}$ gravity with a Higgs scalar field under the requirement of Weyl invariance [12]. Some papers have been devoted to the possible existence and properties of vacuum static, spherically symmetric black holes and wormholes in HMPD [13-15], as well as static cylindrically symmetric configurations that are intended to reproduce the basic properties of cosmic strings [16].

Let us also mention a further generalization of HMPG, containing an arbitrary function of the two curvature scalars $R$ and $\mathcal{R}$, and developed in [17-20].

The present paper is devoted to a study of vacuum static cylindrically symmetric solutions of HMPG. The metric is assumed in the general form

$$
d s^{2}=\mathrm{e}^{2 \gamma} d t^{2}-\mathrm{e}^{2 \alpha} d x^{2}-\mathrm{e}^{2 \mu} d z^{2}-\mathrm{e}^{2 \beta} d \varphi^{2}
$$

where $\alpha, \beta, \gamma, \mu$ are functions of $x ; z \in \mathbb{R}$ and $\varphi \in[0,2 \pi)$ are the longitudinal and azimuthal coordinates, respectively, and the radial coordinate $x$ is arbitrary and admits reparametrization to any smooth monotonic function of $x$. Our goal will be to find regular stringlike configurations, that is, HMPG solutions with the metric (2) having a regular axis and a proper behavior at large values of the circular radius $r \equiv \mathrm{e}^{\beta}$ : the latter means that the metric should be either asymptotically flat or flat up to an angular deficit, which will then be proportional to the effective cosmic string tension. Under these conditions, such a globally regular solitonlike field configuration can be observed from a distant flat or very weakly curved region of space like a cosmic string.

The same objective was formulated in [16], where the authors used the coordinate condition $\alpha=\gamma$ in our notations (that is, $g_{t t}=-g_{x x}$, see Equation (17) in [16], and note that, there, the radial coordinate is denoted $r$ instead of our $x$ ). In addition, based on the desired string interpretation, it was postulated there that the metric should be invariant under boosts in the $z$ direction [21], which leads to the requirement $g_{t t}=-g_{z z}$. As a result, solutions were sought for in [16] under the restrictive condition $\alpha=\gamma=\mu=0$ in the present notations, with only one unknown metric function $\mathrm{e}^{\beta(x)}$ (equal to $W(r)$ in [16], see Equation (29) there). A number of analytical and numerical solutions were then obtained and discussed, but none of them possessed a regular axis and, therefore, none of them were able to represent a regular extended cosmic string model.

In this paper, we undertake an extended study of the same problem. First of all, we do not impose any restriction on the metric (2) (except for a convenient choice of the radial coordinate $x$ ), but show that the requirement of a regular axis and a regular (that is, flat or string) asymptotic behavior inevitably implies $g_{t t}=-g_{z z}$, which leads to boost invariance in the $(t, z)$ subspace and makes it 
unnecessary to separately require this invariance. Furthermore, here we restrict ourselves to seeking only stringlike solutions, formulate some necessary conditions for their existence and present a few examples of such solutions (we will use the terms "stringlike" and "solitonic" as synonyms).

As in [16], we will also employ the scalar-tensor theory (STT) representation of HMPG, but, unlike these authors, we essentially use the well-known conformal mapping leading to the Einstein frame, which formally coincides with GR with a minimally coupled scalar field as the source of gravity. The latter problem has been studied in [22], and a number of results and observations that were obtained there turn out to be useful for studying the present problem. More general data on the necessary mathematical definitons concerning cylindrical space-times and discussions of numerous cylindrically symmetric solutions obtained in GR with different sources of gravity can be found in the book [23] and the review [24].

The paper is organized as follows. In Section 2, we briefly recall the main features of HMPG and its STT representation [6,7]. In Section 3, we formulate the problem to be solved, including the explicit form of the field equations and boundary conditions of regularity at large and small radii. In Section 4, we reproduce the results of [22] that are relevant to our present problem and then use them to obtain some necessary conditions for the existence of stringlike solitonic solutions in HMPG. Section 5 is devoted to particular examples of such solutions, and Section 6 contains a discussion and some concluding remarks.

\section{HMPG and Its Scalar-Tensor Representation}

As is known from $[6,7,13]$, varying the action (1) with respect to the connection $\hat{\Gamma}_{\mu v}^{\alpha}$, we obtain that this connection coincides with the familiar Levi-Civita connection for the metric $h_{\mu \nu}=\phi g_{\mu \nu}$ conformal to $g_{\mu v}$, where the conformal factor is $\phi=F_{\mathcal{R}} \equiv d F / d \mathcal{R}$. It clearly shows that the HMPG actually involves, in addition to $g_{\mu \nu}$, a single additional dynamic degree of freedom that is expressible in the scalar field $\phi$. As is demonstrated in [6,7], the whole theory can be reformulated as a specific example of a scalar-tensor theory (STT) where the gravitational part of the action is

$$
S_{g}=\int d^{4} x \sqrt{-g}\left[(1+\phi) R-\frac{3}{2 \phi}(\partial \phi)^{2}-V(\phi)\right]
$$

where $^{1}$ the potential $V(\phi)$ is expressed in terms of $f(\mathcal{R})$ :

$$
V(\phi)=\mathcal{R} F_{\mathcal{R}}-F(\mathcal{R}) .
$$

The theory (3) belongs to the Bergmann-Wagoner-Nordtvedt class of STT [25-27], characterized by the gravitational action

$$
S_{g}=\int d^{4} x \sqrt{-g}\left[f(\phi) R+h(\phi)(\partial \phi)^{2}-V(\phi)\right]
$$

where $f(\phi), h(\phi)$ and $V(\phi)$ are arbitrary functions. In the present case, $V(\phi)$ is given by (4), and

$$
f(\phi)=1+\phi, \quad h(\phi)=-\frac{3}{2 \phi} .
$$

1 Unlike the papers $[6,7,13]$ etc., we use the metric signature $(+---)$, therefore, the plus before $(\partial \phi)^{2}=g^{\mu v} \phi_{\mu} \phi_{v}$ refers to a canonical field, and a minus means that the field is phantom. Also, in what follows we consider only vacuum HMPG solutions $\left(S_{m}=0\right)$ and safely omit the factor $1 /\left(2 \varkappa^{2}\right)$ near the gravitational part of the action. The Ricci tensor is defined as $R_{\mu \nu}=\partial_{\nu} \Gamma_{\mu \alpha}^{\alpha}-\ldots$, thus, for example, the Ricci scalar $R$ is positive for de Sitter space-time. The units are used in which the speed of light and the Newtonian gravitational constant are equal to unity. 
The general action (5) is known to admit a conformal mapping [26] to the Einstein frame $\mathbb{M}_{E}$ in which the scalar field is minimally coupled to the metric (the formulation (5) is said to correspond to the Jordan conformal frame $\mathbb{M}_{\mathrm{J}}$ ). The transformation is given by [26]

$$
\bar{g}_{\mu \nu}=f(\phi) g_{\mu v}, \quad \frac{d \phi}{d \bar{\phi}}=f(\phi)|D(\phi)|^{-1 / 2}, \quad D(\phi)=f(\phi) h(\phi)+\frac{3}{2}\left(\frac{d f}{d \phi}\right)^{2},
$$

and leads to the action in $\mathbb{M}_{\mathrm{E}}$

$$
S_{g}=\int d^{4} x \sqrt{-\bar{g}}\left[\bar{R}+n \bar{g}^{\mu v} \bar{\phi}_{, \mu} \bar{\phi}_{, v}-\frac{V(\phi)}{f^{2}(\phi)}\right],
$$

where quantities obtained from or with the transformed metric $\bar{g}_{\mu \nu}$ are marked by overbars. The factor $n=\operatorname{sign} D(\phi)$ distinguishes two kinds of scalar fields: $n=+1$ corresponds to canonical scalars with positive kinetic energy, while $n=-1$ describes phantom fields with negative kinetic energy.

In the theory (3), we obtain $D=-3 /(2 \phi)$, so that $n=-\operatorname{sign} \phi$ and, as a result,

$$
\begin{array}{ll}
\phi=-\tanh ^{2} \frac{\bar{\phi}}{\sqrt{6}} & (n=+1,-1<\phi<0), \\
\phi=\tan ^{2} \frac{\bar{\phi}}{\sqrt{6}} \quad(n=-1, \phi>0) .
\end{array}
$$

Thus, according to the sign of $\phi$, the whole theory splits into two sectors, the canonical one and the phantom one. We also notice that values of $\phi$ smaller than -1 are apparently physically meaningless, because they lead to a negative effective gravitational constant.

Substituting $\phi=-n \chi^{2} / 6$ in the action (3), we convert it to

$$
S_{g}=\int d^{4} x \sqrt{-g}\left[\left(1-n \chi^{2} / 6\right) R+n(\partial \chi)^{2}-W(\chi)\right],
$$

where $W(\chi)=V(\phi)$. This action corresponds to GR where the only source of gravity is a conformally coupled scalar field whose kinetic energy has the usual sign if $\phi<0(n=1)$ and the anomalous sign if $\phi>0(n=-1)$. Conformally coupled scalar fields have been studied by many authors, beginning with Penrose [28] (who considered a massless conformally invariant scalar field) and Chernikov and Tagirov [29] (who introduced and analyzed massive conformally coupled fields). The phantom version of (11) was discussed in [30] as a possible alternative to GR in different cosmological and astrophysical applications.

The transition (7) has been quite often used for finding exact or approximate solutions to the STT field equations, because the equations due to (8) are appreciably simpler than those due to the action (5). Having found an Einstein-frame solution, it is easy to obtain its Jordan-frame counterpart while using the transformation inverse to (7).

However, one should bear in mind an important subtle point: if the function $f(\phi)$ in (5) is singular (zero or infinity) at some value of $\phi$, it may happen that a singularity in $\mathbb{M}_{\mathrm{E}}$ with the metric $\bar{g}_{\mu \nu}$ maps

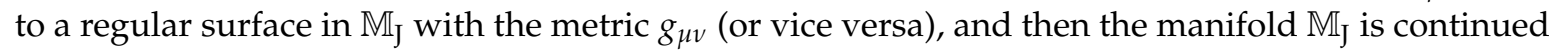
beyond such a surface. This phenomenon has been termed conformal continuation [31,32], and it takes place in many scalar-vacuum and scalar-electrovacuum solutions of STT and $f(R)$ theories of gravity [32,33], including, in particular, spherically symmetric solutions of GR with a conformal scalar field [34,35] and those of the Brans-Dicke theory [36,37]. In the present paper, we will meet conformal continuations while dealing with cylindrically symmetric manifolds.

Using the STT representation, it is natural to ask: if we know a solution with a certain potential $V(\phi)$, what is then the original form of HMPG, in other words, what is the corresponding function $F(\mathcal{R})$ ? The answer follows from Equation (4). In the case $V(\phi) \equiv 0$ we have simply $F(\mathcal{R})=$ const $\cdot \mathcal{R}$. 
In the general case $V(\phi) \not \equiv 0$, since $\phi=F_{\mathcal{R}}$, the relation (4) has the form of a Clairaut equation with respect to $F(\mathcal{R})$ (see, e.g., [38]); its solution consists of a regular family containing only linear functions,

$$
F(\mathcal{R})=H \mathcal{R}-V(H), \quad H=\text { const },
$$

and the so-called singular solution, which forms an envelope of the regular family of solutions and can be written in a parametric form:

$$
\begin{aligned}
& F(\mathcal{R})=\phi \mathcal{R}-V(\phi), \\
& \mathcal{R}=d V / d \phi .
\end{aligned}
$$

A more detailed discussion of this issue can be found in [13].

\section{Cylindrical Symmetry: Equations and Boundary Conditions}

\subsection{Equations}

The final results for HMPG must be formulated in the Jordan frame $\mathbb{M}_{\mathrm{J}}$, for which we assume the metric (2) in slightly different notations,

$$
d s_{J}^{2}=\mathrm{e}^{2 \gamma_{\mathrm{J}}} d t^{2}-\mathrm{e}^{2 \alpha_{\mathrm{J}}} d x^{2}-\mathrm{e}^{2 \mu_{\mathrm{J}}} d z^{2}-\mathrm{e}^{2 \beta_{\mathrm{J}}} d \varphi^{2},
$$

where $\alpha_{\mathrm{J}}, \beta_{\mathrm{J}}, \gamma_{\mathrm{J}}, \mu_{\mathrm{J}}$ are functions of $x$, as well as the scalar field $\phi$. However, we will seek solutions to the field equations in the Einstein frame $\mathbb{M}_{E}$, which corresponds to the action (8), in which, for convenience, we make the substitution $\bar{\phi} \rightarrow \psi$ :

$$
\begin{aligned}
S_{g} & =\int d^{4} x \sqrt{-\bar{g}}\left[\bar{R}+6 n(\bar{\partial} \psi)^{2}-U(\psi)\right], \\
\psi & =\bar{\phi} / \sqrt{6}, \quad(\bar{\partial} \psi)^{2}=\bar{g}^{\mu v} \psi_{, \mu} \psi_{, v}, \quad U(\psi)=\frac{V(\phi)}{(1+\phi)^{2}} .
\end{aligned}
$$

The corresponding field equations read

$$
\begin{aligned}
& 12 n \square \psi+d U / d \psi=0, \\
& \bar{R}_{\mu}^{v}-\frac{1}{2} \delta_{\mu}^{v} \bar{R}=-T_{\mu}^{v}[\psi],
\end{aligned}
$$

where $\square$ is the d'Alambertian operator defined for the metric $\bar{g}_{\mu v}$, and

$$
T_{\mu}^{v}[\psi]=6 n\left[\psi_{, \mu} \psi^{, v}-\frac{1}{2} \delta_{\mu}^{v}(\bar{\partial} \psi)^{2}\right]+\frac{1}{2} \delta_{\mu}^{v} U(\psi) .
$$

These field equations will be considered for the scalar $\psi=\psi(x)$ and the metric

$$
d s_{E}^{2}=\mathrm{e}^{2 \gamma} d t^{2}-\mathrm{e}^{2 \alpha} d x^{2}-\mathrm{e}^{2 \mu} d z^{2}-\mathrm{e}^{2 \beta} d \varphi^{2} .
$$

The Einstein- and Jordan-frame quantities are related by

$$
\begin{array}{ll}
\phi=-\tanh ^{2} \psi, & d s_{J}^{2}=\cosh ^{2} \psi d s_{E}^{2} \quad(n=1, \text { canonical sector }), \\
\phi=\tan ^{2} \psi, & d s_{J}^{2}=\cos ^{2} \psi d s_{E}^{2} \quad(n=-1, \text { phantom sector }) .
\end{array}
$$

It is convenient to use the alternative form of the Einstein equations

$$
\bar{R}_{\mu}^{v}=-6 n \psi, \mu \psi^{\prime v}+\frac{1}{2} \delta_{\mu}^{v} U(\psi) ;
$$


the nonzero components of the Ricci tensor $\bar{R}_{\mu}^{\nu}$ are

$$
\begin{aligned}
& \bar{R}_{0}^{0}=-\mathrm{e}^{-2 \alpha}\left[\gamma^{\prime \prime}+\gamma^{\prime}\left(\sigma^{\prime}-\alpha^{\prime}\right)\right], \\
& \bar{R}_{1}^{1}=-\mathrm{e}^{-2 \alpha}\left[\sigma^{\prime \prime}+\sigma^{\prime 2}-2\left(\beta^{\prime} \gamma^{\prime}+\beta^{\prime} \mu^{\prime}+\gamma^{\prime} \mu^{\prime}\right)-\alpha^{\prime} \sigma^{\prime}\right], \\
& \bar{R}_{2}^{2}=-\mathrm{e}^{-2 \alpha}\left[\mu^{\prime \prime}+\mu^{\prime}\left(\sigma^{\prime}-\alpha^{\prime}\right)\right], \\
& \bar{R}_{3}^{3}=-\mathrm{e}^{-2 \alpha}\left[\beta^{\prime \prime}+\beta^{\prime}\left(\sigma^{\prime}-\alpha^{\prime}\right)\right],
\end{aligned}
$$

where the prime denotes $d / d x$ and we have introduced the notation

$$
\sigma=\beta+\gamma+\mu
$$

it is also helpful to write the constraint equation from (17), which is the first integral of the others and only contains first-order derivatives of the metric functions:

$$
\beta^{\prime} \gamma^{\prime}+\beta^{\prime} \mu^{\prime}+\gamma^{\prime} \mu^{\prime}=3 n \psi^{\prime 2}-\frac{1}{2} U(\psi) \mathrm{e}^{2 \alpha}
$$

\subsection{Boundary Conditions}

Let us, for convenience, formulate the boundary conditions in the Einstein frame, referring to their more detailed description in [22]. Similar conditions for $\mathbb{M}_{J}$ will only require putting the index "J" near each of the letters $\alpha, \beta, \gamma, \mu$.

A regular axis means that there is some value $x=x_{\mathrm{ax}}$ at which the circular radius $r=\mathrm{e}^{\beta} \rightarrow 0$ while the algebraic curvature invariants remain finite. Let us note that the Kretschmann invariant $K=R^{\mu v \rho \sigma} R_{\mu \nu \rho \sigma}$ for the metric (19) is a sum of squared components of the nonzero Riemann tensor components $R_{\rho \sigma}^{\mu v}$ :

$$
\begin{aligned}
K & =4 \sum_{i=1}^{6} K_{i}^{2} ; \\
K_{1} & =R^{01}{ }_{01}=-\mathrm{e}^{-\alpha-\gamma}\left(\gamma^{\prime} \mathrm{e}^{\gamma-\alpha}\right)^{\prime}, \\
K_{2} & =R^{02}{ }_{02}=-\mathrm{e}^{-2 \alpha} \gamma^{\prime} \mu^{\prime}, \\
K_{3} & =R^{03}{ }_{03}=-\mathrm{e}^{-2 \alpha} \beta^{\prime} \gamma^{\prime}, \\
K_{4} & =R^{12}{ }_{12}=-\mathrm{e}^{-\alpha-\mu}\left(\mu^{\prime} \mathrm{e}^{\mu-\alpha}\right)^{\prime}, \\
K_{5} & =R^{13}{ }_{13}=-\mathrm{e}^{-\alpha-\beta}\left(\beta^{\prime} \mathrm{e}^{\beta-\alpha}\right)^{\prime}, \\
K_{6} & =R^{23}{ }_{23}=-\mathrm{e}^{-2 \alpha} \beta^{\prime} \mu^{\prime}
\end{aligned}
$$

Thus, for $K<\infty$ it is necessary and sufficient that all $\left|K_{i}\right|<\infty$, which in turn guarantees finite values of all algebraic invariants composed from the Riemann tensor. It is important that all $K_{i}$ in (26) are independent of the choice of the coordinate $x$.

One can verify [22] that all $K_{i}$ are finite on the axis $r=\mathrm{e}^{\beta} \rightarrow 0$ if and only if $\gamma(x)$ and $\mu(x)$ tend to finite limits $\gamma_{\mathrm{ax}}$ and $\mu_{\mathrm{ax}}$, and

$$
\begin{aligned}
& \gamma^{\prime} \mathrm{e}^{-\alpha}=O(r), \quad \mu^{\prime} \mathrm{e}^{-\alpha}=O(r), \\
& \left|\beta^{\prime}\right| \mathrm{e}^{\beta-\alpha}=1+O\left(r^{2}\right) .
\end{aligned}
$$


the latter condition expressing a correct relation between an infinitesimal circumference and its radius, in other words, the absence of a conical singularity on the axis ${ }^{2}$.

A correct asymptotic behavior, guaranteeing that our configuration will be visible for a distant observer, requires a zero curvature limit, which, in turn, implies finite values of $\gamma(x)$ and $\mu(x)$ at some $x=x_{\infty}$, where $r=\mathrm{e}^{\beta} \rightarrow \infty$. Moreover, a flat-space limit at spatial infinity would require a condition that is similar to (28), but a cosmic string geometry admits a more general condition,

$$
\left|\beta^{\prime}\right| \mathrm{e}^{\beta-\alpha} \rightarrow 1-\mu_{s}, \quad \mu_{s}=\text { const }<1,
$$

where $2 \pi \mu_{s}$ is the angular deficit that characterizes the gravitational field of a cosmic string. Thus, the space-time is locally flat, but globally behaves as if there were a conical singularity. Following [22], we will call such a flat or stringlike asymptotic behavior at large $\mathrm{e}^{\beta}$ "a regular asymptotic".

Under the above regularity conditions on the axis and at infinity, the total energy of matter per unit length along the $z$ axis is finite, as can be easily verified (for example, by using the Gaussian normal radial coordinate specified by the condition $\mathrm{e}^{\alpha} \equiv 1$ ). This quantity is determined by the integral

$$
\mathcal{E}=\int T_{0}^{0} \mathrm{e}^{\alpha+\beta+\mu} d x d z d \varphi
$$

(where integration in $z$ covers a unit interval), which converges at infinity, because the Ricci tensor components (23) decay there as $r^{-3}$ or faster (assuming that all quantities can be expanded in power series in $1 / r)$, and the same is true for $T_{\mu}^{\nu}$ due to the Einstein equations. Recalling the expression (18) for $T_{\mu}^{v}$, we conclude that, at a regular asymptotic, both quantities $U$ and $\mathrm{e}^{-2 \alpha} \psi^{\prime 2}$ decay at infinity as $r^{-3}$ or faster.

The angular deficit $2 \pi \mu_{s}$ is directly proportional to the quantity (30) in the simplest string model with a flat metric everywhere except the symmetry axis, where a conical singularity takes place [21]. Such a relationship is not evident in the general case.

We are seeking solitonic solutions that possess both a regular axis and a regular asymptotic.

\section{Stringlike Solutions: Analysis}

\subsection{Solitons in the Einstein Frame}

In this subsection, we briefly reproduce and discuss some results of [22], relevant to our study, and add some more observations.

Let us choose the harmonic $x$ coordinate in the metric (19), such that ${ }^{3}$

$$
\alpha=\beta+\gamma+\mu
$$

(note that such a condition does not hold in the corresponding Jordan-frame metrics).

Using the important property of any scalar fields minimally coupled to gravity,

$$
T_{0}^{0}=T_{2}^{2}=T_{3}^{3},
$$

and the expressions (23), it is easy to find that Equation (22) combine to give

$$
\beta^{\prime \prime}=\gamma^{\prime \prime}=\mu^{\prime \prime}=\frac{1}{3} \alpha^{\prime \prime}
$$

2 We denote by $O(f)$ a quantity of either the same order of magnitude as $f$ or smaller in a certain limit, while quantities of the same order are connected by the symbol $\sim$.

3 To our knowledge, this coordinate condition was used for the first time for finding cylindrically symmetric solutions in GR in [39]. 
(where the last equality holds due to (31)), whence it follows

$$
\begin{aligned}
& \mu=\frac{1}{3}(\alpha-A x) \\
& \gamma=\frac{1}{3}(\alpha-B x) \\
& \beta=\frac{1}{3}(\alpha+A x+B x)
\end{aligned}
$$

where $A$ and $B$ are integration constants; other two constants are ruled out by properly choosing the origin of the $x$ coordinate and the scale along the $z$ axis.

The remaining equations for the unknowns $\alpha(x)$ and $\psi(x)$ read

$$
\begin{aligned}
& 2 \alpha^{\prime \prime}=-3 U \mathrm{e}^{2 \alpha}, \\
& 12 n \psi^{\prime \prime}=\mathrm{e}^{2 \alpha} d U / d \psi, \\
& \alpha^{\prime 2}-9 n \psi^{\prime 2}=-\frac{3}{2} U \mathrm{e}^{2 \alpha}+\frac{1}{3}\left(A^{2}+A B+B^{2}\right),
\end{aligned}
$$

where Equation (37) follows from (25) and is a first integral of (35) and (36).

A value of $x$ where $\beta \rightarrow-\infty$, so that coordinate circles shrink to a point, corresponds to an axis, whereas spatial infinity corresponds to $\beta \rightarrow \infty$. In the coordinates (31) the conditions (27), (28), or those of a regular asymptotic can only hold at $x \rightarrow \pm \infty$. Because $\mu$ and $\gamma$ must, there, tend to finite limits simultaneously, from (34) it follows that a regular axis (say, at $x \rightarrow-\infty$ ) or a regular asymptotic (at $x=+\infty$ ) can exist if the integration constants satisfy the requirement

$$
A=B=N>0
$$

Thus, a regular axis and a regular asymptotic require the same relation (38), which is favorable for the existence of solitonic solutions.

Suppose that there is a solitonic solution, regular at $x \rightarrow \pm \infty$. Consequently, at both ends we have in the leading order of magnitude in $x$

$$
\alpha(x) \approx \beta(x) \approx N x
$$

At infinity, $x \rightarrow \infty$, choosing the $t$ - and $z$-scales so that $\gamma=\mu \rightarrow 0$, we can write

$$
\alpha(x)=\beta(x)+o(1) \approx N x
$$

hence, the constant $N$ has a clear geometric meaning: according to (29),

$$
N=1-\mu_{s}
$$

where $2 \pi \mu_{s}$ is the angular deficit at a string asymptotic.

On the regular axis $x \rightarrow-\infty$, since the $t$ - and $z$-scales have already been fixed by the conditions at infinity, we can only assume $\gamma=\mu \rightarrow \gamma_{\mathrm{ax}}=$ const, hence, recalling the coordinate condition (31), the regularity condition (28), $\mathrm{e}^{\beta-\alpha} \beta^{\prime} \rightarrow 1$, leads to

$$
\mathrm{e}^{-2 \gamma_{\mathrm{ax}}} N=1 \Rightarrow \mathrm{e}^{2 \gamma_{\mathrm{ax}}}=N=1-\mu_{\mathrm{s}}
$$

It means that the value of the "redshift function" $g_{t t}=\mathrm{e}^{2 \gamma}$ on the axis is directly related to the angular deficit at infinity.

There is one more point of importance to bear in mind: in accord with (27) and to provide finiteness of $T_{\mu}^{\nu}$ on the axis, it is necessary to require

$$
\left|\alpha^{\prime \prime}\right| \mathrm{e}^{-2 \alpha}<\infty \text { as } x \rightarrow-\infty
$$


from which it directly follows $|U|<\infty$ by (35). At large positive $x$ no additional requirements are needed, we already have there $U \rightarrow 0$ due to (40).

As $x \rightarrow \infty$, we must also have $\mathrm{e}^{-2 \alpha} \psi^{\prime 2} \rightarrow 0$ as a necessary condition of vanishing curvature. The behavior of the scalar field $\psi$ is closely related to that of $\alpha$ since, excluding $U$ from (37) and (35) and, using (38), we obtain

$$
9 n \psi^{\prime 2}=\alpha^{2}-\alpha^{\prime \prime}-N^{2} .
$$

In particular, on the axis $x \rightarrow-\infty$, we can assume $\alpha^{\prime}=N+O(r)=N+O\left(\mathrm{e}^{N x}\right)$; hence, due to (44), we have $\psi^{\prime}=O\left(\mathrm{e}^{-N|x| / 2}\right)$ leading to a finite value of $\psi(-\infty)$. On the other hand, the requirement of a sufficiently fast decay of $\mathrm{e}^{-2 \alpha} \psi^{\prime 2}$ as $x \rightarrow \infty$ (see the end of Section 3 ) leads to a finite value of $\psi(+\infty)$. Thus the $\psi$ field should vary in a finite range over all $x \in \mathbb{R}$.

Furthermore, integrating Equation (35) over all $x$, as long as $\alpha^{\prime}( \pm \infty)=N$, we obtain

$$
\int_{-\infty}^{+\infty} U(\psi(x)) \mathrm{e}^{2 \alpha} d x=0
$$

which means that $U(\psi) \neq 0$ has a variable sign in any solitonic solution.

Another important observation is that, due to $A=B$, we have $\mu \equiv \gamma$, that is, $\bar{g}_{z z}=\bar{g}_{t t}$, hence any solitonic solution in $\mathbb{M}_{\mathrm{E}}$ is boost-invariant in the $(z, t)$ subspace.

\subsection{Solitons in the Jordan Frame}

Let us find out how the above observations are modified if we seek a solitonic solution in the Jordan frame $\mathbb{M}_{\mathrm{J}}$. For its description, we can use the same functions $\psi, \alpha, \beta, \gamma, \mu$ as in $\mathbb{M}_{\mathrm{E}}$, obeying the same equations, but the boundary conditions should now be formulated for the metric (14).

We can write for any STT from the class (5)

$$
d s_{J}^{2}=\mathrm{e}^{2 \eta(x)} d s_{E}^{2}
$$

the function $\eta(x)$ depending on the choice of a theory. Therefore, we have for the metrics (14) and (19) $\alpha_{\mathrm{J}}=\alpha+\eta$ and similarly for other metric coefficients. Consider the regular asymptotic requirements in $\mathbb{M}_{\mathrm{J}}$, then, at the corresponding value $x=x_{\infty}$, we should have finite values of

$$
\gamma_{\mathrm{J}}=\gamma+\eta \quad \text { and } \quad \mu_{\mathrm{J}}=\mu+\eta
$$

and an infinite value of $\beta_{\mathrm{J}}=\beta+\eta$. Because Equation (34) hold as before, the above requirements can be satisfied only if $x_{\infty}= \pm \infty$ (let it be $+\infty$ without loss of generality), and $\mu-\gamma$ should be finite in this limit. From (34), it then follows that $A=B=N>0$ and $\mu \equiv \gamma$. We thus come to a similar conclusion to the one made for solitonic solutions in $\mathbb{M}_{\mathrm{E}}$, now that $g_{t t}=-g_{z z}$. Even more than that: the same could be concluded if we required a regular axis instead of a regular asymptotic (where, for consistency, we will put $\left.x=x_{\mathrm{ax}}=-\infty\right)$. We can state the following:

In any STT from the class (5), a static vacuum cylindrically symmetric solution with a regular asymptotic and/or regular axis is necessarily boost-invariant in the $(z, t)$ subspace.

Thus, it is unnecessary to postulate this stringy property [21], since it directly follows from the proper boundary conditions.

Next, we can compare two expressions for $\beta$ : one obtained from (34), with $A=B=N>0$, that is, $\beta=\frac{1}{3}(\alpha+2 N x)$, and the other, following from (31): $\beta=\alpha-\gamma-\mu=\alpha-\gamma_{\mathrm{J}}-\mu_{\mathrm{J}}+2 \eta$. Since $\gamma_{\mathrm{J}}=\mu_{\mathrm{J}}$ tend to finite limits as $x \rightarrow \infty$, this comparison gives in the same limit:

$$
\alpha=N x-3 \eta+O(1), \quad \beta=N x-\eta+O(1) .
$$


Let us now apply the boundary condition (29) in $\mathbb{M}_{\mathrm{J}}$ : as $x \rightarrow \infty$,

$$
\mathrm{e}^{\beta_{\mathrm{J}}-\alpha_{\mathrm{J}}} \beta_{\mathrm{J}}^{\prime} \sim \mathrm{e}^{2 \eta}\left(\beta^{\prime}+\eta^{\prime}\right) \sim \mathrm{e}^{2 \eta} N=O(1),
$$

whence it follows that $\eta(x)$ tends to a finite limit at large $x$. As a result, we have finite limits of the Einstein-frame quantities $\gamma$ and $\mu$ and also $\alpha \sim \beta \sim N x$, in full similarity with the Einstein frame. In (48), we have used the fact that $\eta^{\prime}=(d \eta / d \psi) \psi^{\prime}$ vanishes as $x \rightarrow \infty$, since $\psi^{\prime} \rightarrow 0$ (see (44)), while $d \eta / d \psi$ must be finite due to the the regularity requirement for $\eta(\psi)$.

Quite a similar reasoning can be used for $x \rightarrow-\infty$ (the axis), with the difference that now it is required $\mathrm{e}^{\beta_{\mathrm{J}}-\alpha_{\mathrm{J}}} \beta_{\mathrm{J}}^{\prime} \rightarrow 1$.

So far, in this subsection, we did not make any assumptions on the properties of a solution in $\mathbb{M}_{\mathrm{E}}$ and even did not use the evident requirement that $\eta(\psi)$ should be regular in the whole range $x \in \mathbb{R}$, considering each limit $x \rightarrow \pm \infty$ separately and using the transformation (7) as simply a substitution in the field equations. Invoking the boundary conditions, we have concluded that $\eta(\psi)$ must not only be regular, but also must have finite limits on the axis and at infinity.

We can now assert that in the limit $x \rightarrow-\infty$ we have finite values of $\eta=\eta_{\mathrm{ax}}, \mu=\gamma=\gamma_{\mathrm{ax}}$ as well as $\beta_{\mathrm{J}}-\alpha_{\mathrm{J}}=\beta-\alpha$; in addition, $\eta^{\prime} \rightarrow 0$, therefore, in this limit,

$$
\mathrm{e}^{\beta_{\mathrm{J}}-\alpha_{\mathrm{J}}} \beta_{\mathrm{J}}^{\prime}=\mathrm{e}^{\beta-\alpha} \beta^{\prime}=1,
$$

as required in (28). Thus, a regular axis in $\mathbb{M}_{\mathrm{J}}$ implies a regular axis in $\mathbb{M}_{\mathrm{E}}$.

At the asymptotic $x \rightarrow+\infty$, the first equality of (49) again holds, which means that a regular

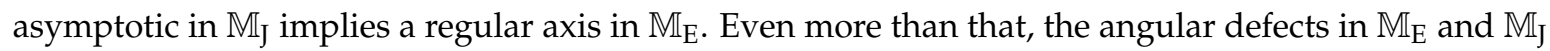
coincide, $\mu_{s}=1-N$. What is different is the relationship between $N$ and the "redshift function" $\mathrm{e}^{2 \gamma_{\mathrm{J}}}$ : since $\gamma_{\mathrm{J}}=\gamma+\eta$, we now have

$$
\left.\mathrm{e}^{2 \gamma_{\mathrm{J}}}\right|_{x \rightarrow-\infty}=N \mathrm{e}^{2 \eta_{\mathrm{ax}}}
$$

Thus, we have the following general result:

If the conformal factor $\mathrm{e}^{2 \eta}$ is finite in the whole range $x \in \mathbb{R}$ and in the limits $x \rightarrow \pm \infty$, then solitonic solutions exist simultaneously in $\mathbb{M}_{\mathrm{J}}$ and in $\mathbb{M}_{\mathrm{E}}$, and they are characterized by the same deficit angle.

A possible singular behavior of the conformal factor violates this correspondence, as is evident from the example (21): if we have a solitonic solution in $\mathbb{M}_{\mathrm{E}}$ but $\cos \psi=0$ at some $x$, on such a surface we have a singularity in $\mathbb{M}_{\mathrm{J}}$. It is an evident case of a conformal continuation, in which the transformation (7) maps the whole singular manifold $\mathbb{M}_{\text {J }}$ to only a part of the regular manifold $\mathbb{M}_{\mathrm{E}}$.

We also cannot exclude a contrary situation, that there is a solitonic solution in $\mathbb{M}_{J}$ but this manifold (or its part) maps to a singular manifold $\mathbb{M}_{\mathrm{E}}$, similarly to what is described, for example, in [35], where $\mathbb{M}_{\mathrm{J}}$ is a wormhole with a conformal scalar, but only its region maps to a singular (Fisher) space-time $\mathbb{M}_{\mathrm{E}}$.

The important constraint (45) on the scalar field potential obtained in $\mathbb{M}_{\mathrm{E}}$ easily transforms to $\mathbb{M}_{\mathrm{J}}$ : indeed, according to (7), we find that $U(\psi)=\mathrm{e}^{4 \eta} V(\phi)$ and $\mathrm{e}^{2 \alpha}=\mathrm{e}^{2 \alpha_{\mathrm{J}}-2 \eta}$ and rewrite Equation (45) in terms of the quantities specified in $\mathbb{M}_{\mathrm{J}}$ as

$$
\int_{-\infty}^{+\infty} V(\phi) \mathrm{e}^{2 \alpha_{\mathrm{J}}+2 \eta} d x=0
$$

where $x$ is, as before, a harmonic coordinate in $\mathbb{M}_{E}$.

The whole content of this subsection applies to any STT from the class (5). 


\section{Examples}

The first step in attempts to find solitons in $\mathbb{M}_{\mathrm{J}}$ as described in the previous section, is to solve the set of equations

$$
\begin{aligned}
& 2 \alpha^{\prime \prime}=-3 U \mathrm{e}^{2 \alpha}, \\
& 12 n \psi^{\prime \prime}=\mathrm{e}^{2 \alpha} d U / d \psi, \\
& \alpha^{\prime 2}-9 n \psi^{\prime 2}=N^{2}-\frac{3}{2} U \mathrm{e}^{2 \alpha},
\end{aligned}
$$

with the unknowns $\alpha(x)$ and $\psi(x)$, constants $n= \pm 1$ and $N>0$, while the choice of the potential $U(x)$ corresponds to the choice of a particular HMPG theory. A combination of (52) and (54) free from $U$ is

$$
9 n \psi^{\prime 2}=\alpha^{\prime 2}-\alpha^{\prime \prime}-N^{2} .
$$

There are some examples of $U(x)$ with which Equations (52)-(54) can be analytically solved, but, in general, with given $U(\psi)$, numerical methods are necessary. An alternative way is to use inverse problem methods [22], of which the simplest is to specify the function $\alpha(x)$ or $\alpha^{\prime}(x)$ and to find $\psi(x)$ and $U(x)$ from the equations; the function $U(\psi)$ is then well defined if $\psi(x)$ is monotonic. An advantage of this method is that, from the previous analysis, we know much about the behavior of $\alpha(x)$ suitable for a solitonic solution.

The following algorithm can be suggested for finding a solitonic solution in $\mathbb{M}_{\mathrm{j}}$ :

1. Specify $\alpha(x)$ regular in $x \in \mathbb{R}$ and such that $\alpha^{\prime}( \pm \infty)=N>0$.

2. Verify the suitable behavior of $\alpha(x)$ : that

(i) $\left|\alpha^{\prime \prime}\right| \mathrm{e}^{-2 \alpha}<\infty$ as $x \rightarrow-\infty$ (see (43)) and (ii) that the r.h.s. of Equation (55) has a definite sign at all $x \in \mathbb{R}$ (we obtain $n=1$, the canonical sector, if it is nonnegative, and $n=-1$ otherwise).

3. Find $\psi(x)$ from Equation (55).

4. Verify that the range of $\psi$ is within the validity range of the map (7).

5. Find $U(x)$ from Equation (52).

6. With the solution found, verify the validity of Equation (53).

Step 2(ii) is necessary as long as we adhere to systems with either a canonical or a phantom scalar field, excluding transitions from one sector to another within a single solution (such solutions do exist and have been considered as possible sources for wormholes with so-called "trapped ghosts" [40-42], this name used because a phantom field exists there in a bounded part of space).

For step 4, it is important that only derivatives of $\psi$ appear in Equations (52)-(55); therefore, one can add to $\psi$ an arbitrary constant to adjust its range to that of the map (7).

The last step is needed only for confidence, since (53) is a consequence of (52) and (54).

\subsection{Example 1: Zero Potential}

This example is trivial, it does not promise any solitonic configurations, and we only present it here for completeness and clarity. The corresponding solution in $\mathbb{M}_{\mathrm{E}}$ is well known, it is a direct extension of the famous century-old Levi-Civita solution [24,43]. In our coordinates and notations, the whole set of equations in $\mathbb{M}_{\mathrm{E}}$ reduces to

$$
\begin{aligned}
& \psi^{\prime \prime}=\beta^{\prime \prime}=\gamma^{\prime \prime}=\mu^{\prime \prime}=0, \\
& \beta^{\prime} \gamma^{\prime}+\beta^{\prime} \mu^{\prime}+\gamma^{\prime} \mu^{\prime}=3 n \psi^{\prime 2},
\end{aligned}
$$


and its solution can be written, without a loss of generality, as

$$
\begin{gathered}
d s_{E}^{2}=\mathrm{e}^{2 c x} d t^{2}-\mathrm{e}^{2 a x} d x^{2}-\mathrm{e}^{2 m x} d z^{2}-\mathrm{e}^{2 b x} d \varphi^{2}, \\
\psi=\psi_{0}+C x, \quad b c+b m+c m=3 n C^{2},
\end{gathered}
$$

where $b, c, m, \psi_{0}, \psi_{1}$ are integration constants and $a=b+c+m$. The requirement of flatness at large $x$ leads to $c=m=0$; hence, by (58), we have $\psi_{1}=0$ (the scalar field is trivial) and a Minkowski metric up to an angular deficit $\mu_{s}=1-b$. The metric is globally flat if $b=1$ and has a conical singularity on the axis $(x \rightarrow-\infty)$ if $b \neq 1$.

\subsection{Example 2: Exponential Potential}

Assuming the potential in the form

$$
U(\psi)=U_{0} \mathrm{e}^{\lambda \psi}, \quad U_{0}, \lambda=\text { const },
$$

it is easy to solve Equations (52)-(54) analytically, but it is impossible to obtain a stringlike solution because of a constant sign of $U$, recall (45).

Indeed, with (59), combining Equations (52) and (53), we obtain

$$
\lambda \alpha^{\prime \prime}+18 n \psi^{\prime \prime}=0 \Rightarrow \lambda \alpha^{\prime}+18 n \psi^{\prime}=C,
$$

where the integration constan $C$ can be fixed from the regular axis conditions: since, as we know, $\alpha^{\prime} \rightarrow N$ and $\psi^{\prime} \rightarrow 0$ as $x \rightarrow-\infty$, we have $C=\lambda N$.

Substituting (60) with $C=\lambda N$ to (54), we obtain

$$
\alpha^{\prime 2}-\frac{n \lambda^{2}}{36}\left(N-\alpha^{\prime}\right)^{2}-N^{2}=\frac{3}{2} U_{0} \mathrm{e}^{\lambda \psi+2 \alpha} .
$$

At a regular asymptotic $x \rightarrow \infty$ we must have $\alpha^{\prime} \rightarrow N$, so that the 1.h.s of Equation (61) turns to zero, whereas the r.h.s. tends to infinity due to $\alpha \approx N x$, while $\psi$ should tend to a finite constant. This contradiction shows that, assuming a regular axis, it is impossible to obtain a regular asymptotic.

Therefore, we will stop here, even though Equation (61) can be easily further integrated (see, e.g., [22] for a solution in slightly different notations). Let us only note that our observations on the exponential potential include the special case of a constant potential, $\lambda=0$ in (59), equivalent to a cosmological constant in $\mathbb{M}_{\mathrm{E}}$.

\subsection{Example 3: Solitons with Nonzero Potential on the Axis}

The calculations turn out to be easier if we specify $\alpha^{\prime}$ as a function of $\alpha$ instead of $\alpha(x)$. The following example uses a suggestion from [22], where it was discussed in the framework of GR: we suppose

$$
\alpha^{\prime 2}=N^{2}\left(1+\frac{H}{\cosh ^{2}(k \alpha)}\right), \quad k=\text { const }>0, \quad H=\text { const }>-1 .
$$

From (62), with (55), it is easy to obtain

$$
\begin{aligned}
& \frac{d}{d \alpha}\left(\alpha^{\prime 2}\right)=2 \alpha^{\prime \prime}=-\frac{2 H N^{2} k \sinh (k \alpha)}{\cosh ^{3}(k \alpha)}, \\
& 9 n \psi^{\prime 2}=\frac{H N^{2}}{\cosh ^{2}(k \alpha)}(1+k \tanh (k \alpha)),
\end{aligned}
$$


where, as before, the prime denotes $d / d x$.

From (64) it follows that to keep the same sign of $n \psi^{\prime 2}$ for all $\alpha$ (and $x$ as well) we should require $k \leq 1$. On the other hand, on the axis $x \rightarrow-\infty$, we have $\alpha \sim N x \rightarrow-\infty$, and by (64) $\alpha^{\prime \prime} \sim \mathrm{e}^{-2 k|\alpha|}$, therefore, to have a finite limit of $\alpha^{\prime \prime} \mathrm{e}^{-2 \alpha}$ according to (43) it is necessary to require $k \geq 1$. Thus, we arrive at the unambiguous value $k=1$.

Putting $k=1$, by direct calculation of $x=\int d \alpha / \alpha^{\prime}$ with (62), we obtain without loss of generality

$$
\sinh (N x)=\frac{\sinh \alpha}{\sqrt{1+H}}
$$

thus, as required, $\alpha \in \mathbb{R}$, and its infinities coincide with those of $x$. The potential $U(x)$ is determined by (52) and, hence, by (63).

We deal with the canonical sector if $H>0$ and with the phantom sector if $H<0$. For calculations, it is more convenient to pass on in (64) from $\psi^{\prime}$ to $\psi_{\alpha} \equiv d \psi / d \alpha=\psi^{\prime} / \alpha^{\prime}$ :

$$
9 n \psi_{\alpha}^{2}=9 n \frac{\psi^{\prime 2}}{\alpha^{\prime 2}}=\frac{H(1+\tanh \alpha)}{H+\cosh ^{2} \alpha} .
$$

In all cases, we see that at large $|\alpha|$ the expression for $\psi^{\prime}$ vanishes exponentially; hence, $\psi$ has finite limits $\psi( \pm \infty)=\psi_{ \pm}$. Thus, in the canonical sector, where the conformal factor $\mathrm{e}^{2 \eta}=\cosh ^{2} \psi$ is specified for $\psi \in R$, we certainly obtain a family of solitonic solutions in $\mathbb{M}_{\mathrm{J}}$, while, in the phantom sector $\left(\mathrm{e}^{2 \eta}=\cos ^{2} \psi\right)$, we must select such parameter values that the range of $\psi$ is located within a single half-wave of $\cos \psi$. Let us discuss them separately and graphically illustrate the behavior of solitonic solutions for selected values of the parameters.

\subsubsection{The Canonical Sector, $n=1$}

Let us put for certainty $H=1 / 2$. Using (65) and (55), the function $\psi(x)$ for given values of $N$ is determined by the integral

$$
\psi(x)=\int_{0}^{x} \sqrt{\frac{\alpha^{\prime}(\xi)^{2}-\alpha^{\prime \prime}(\xi)-N^{2}}{9 n}} d \xi
$$

and is plotted in Figure 1 for selected values of N. Furthermore, using the expressions

$$
U=-\frac{2}{3} \alpha^{\prime \prime} \mathrm{e}^{-2 \alpha}, \quad r=\exp \left(\frac{\alpha+2 N x}{3}\right)
$$

and the relation (65), we plot the potential $U$ as a function of the circular radius $r=\mathrm{e}^{\beta}$ in Figure 2 .

In order to obtain a similar picture for the potential $V$ in $\mathbb{M}_{\mathrm{J}}$, we use relations between the potentials and the circular radii $r$ and $r_{\mathrm{J}}=\mathrm{e}^{\beta_{\mathrm{J}}}$ in the two frames:

$$
\begin{aligned}
& V=(1+\phi)^{2} U=-\frac{2}{3} \cosh ^{-4} \psi \alpha^{\prime \prime} \mathrm{e}^{-2 \alpha} \\
& r_{\mathrm{J}}=r \cosh \psi=\exp \left(\frac{\alpha+2 N x}{3}\right) \cosh \psi
\end{aligned}
$$

The results for the same parameter values as in Figures 1 and 2 can be seen in Figure 3, and they are qualitatively similar to those in Figure 2. 


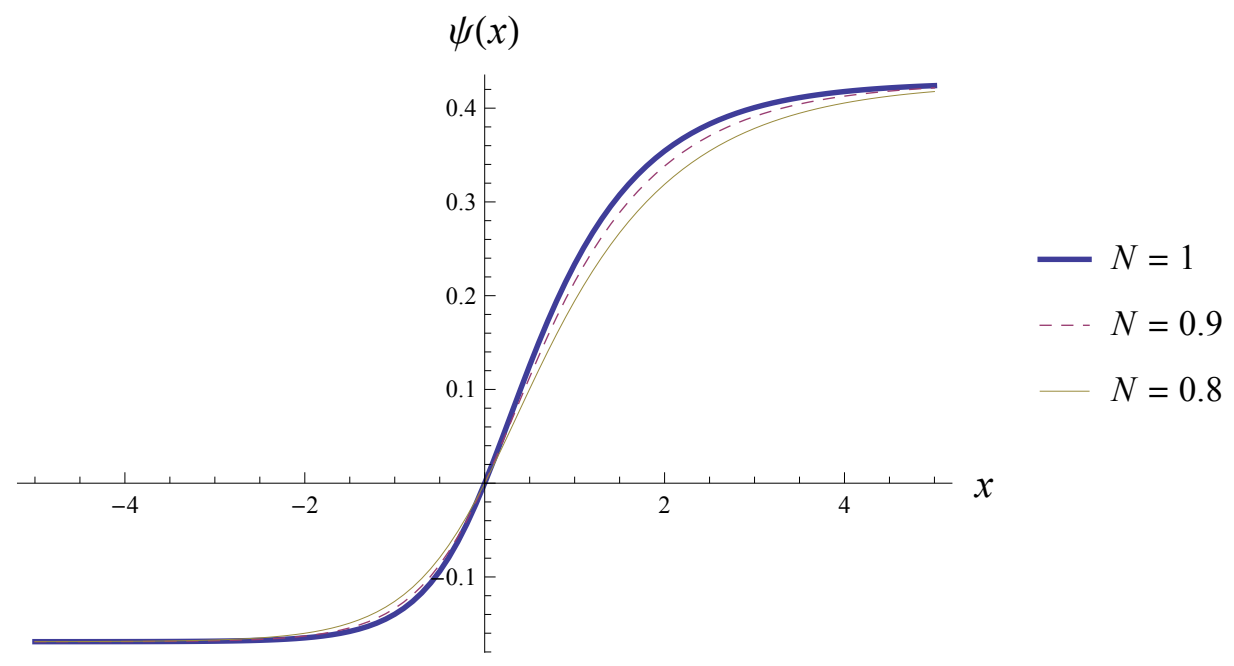

Figure 1. Plots of $\psi(x)$ for $H=1 / 2$ (the canonical sector) and $N=1,0.9,0.8$.

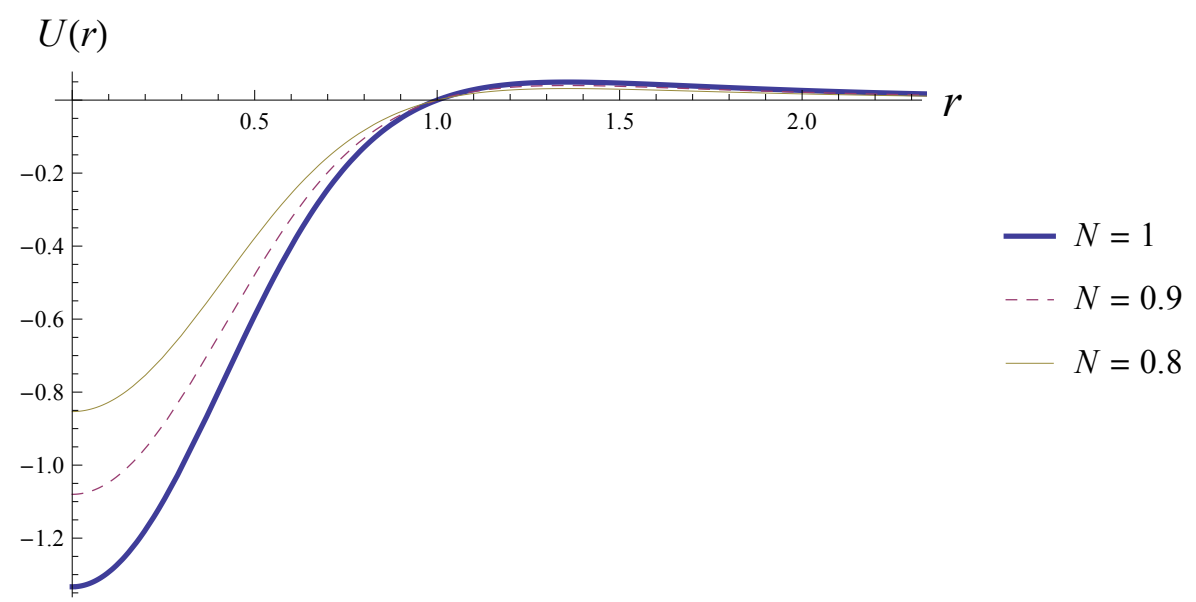

Figure 2. The potential $U(r)$ in the Einstein frame for $H=1 / 2$ and $N=1,0.9,0.8$.

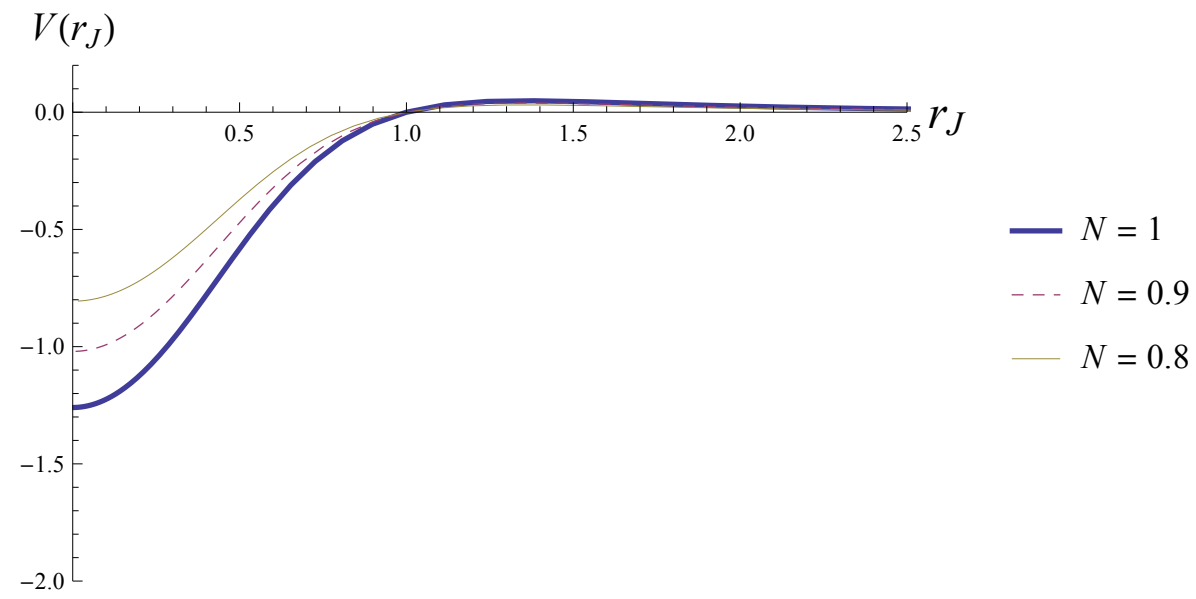

Figure 3. The potential $V\left(r_{\mathrm{J}}\right)$ in the Jordan frame for $H=1 / 2$ and $N=1,0.9,0.8$. 


\subsubsection{The Phantom Sector, $n=-1$}

In this case we can put $H=-1 / 2$. Using the same methodology as for the canonical sector, we plot $\psi(x)$ obtained from (67) and (65) in Figure 4.

The range of $\psi(x)$, coinciding with the difference between its asymptotic values,

$$
\Delta \psi_{ \pm}=\psi(+\infty)-\psi(-\infty) \approx 0.795<\pi,
$$

is located within a single half-wave of $\cos \psi$, as required.

The potential $U(r)$ in the Einstein frame is obtained in the same way as for the canonical sector and it is plotted in Figure 5. In order to obtain the potential $V\left(r_{\mathrm{J}}\right)$ in $\mathbb{M}_{\mathrm{J}}$, we use the following expressions:

$$
\begin{aligned}
& V=(1+\phi)^{2} U=-\frac{2}{3 \cos ^{4} \psi} \alpha^{\prime \prime} \mathrm{e}^{-2 \alpha}, \\
& r_{\mathrm{J}}=r|\cos \psi|=\exp \left(\frac{\alpha+2 N x}{3}\right)|\cos \psi| .
\end{aligned}
$$

Figure 6 shows the corresponding parametric plots.

It is easy to notice that all potentials in the stringlike solutions have a changing sign, in agreement with (45) and (51). Additionally, in the canonical sector, the potential close to the axis has an attracting nature. In the phantom sector it looks repulsive, but it is still attractive for a phantom field, which, in general, tends to climb a potential instead of rolling down.

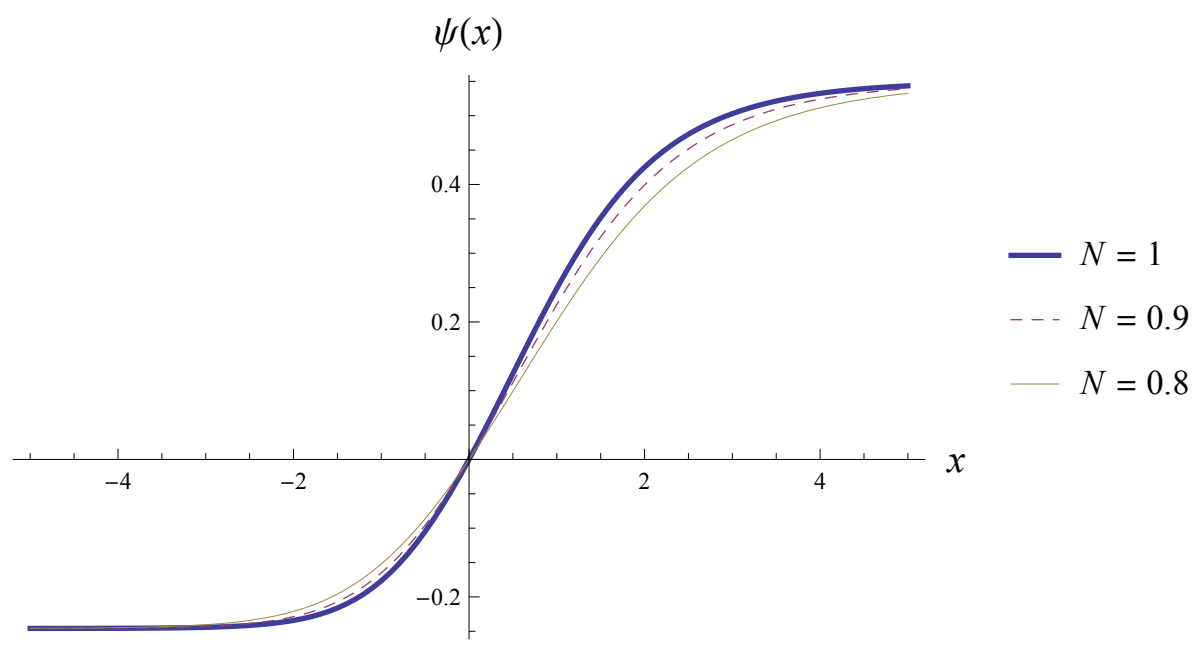

Figure 4. The scalar field $\psi(x)$ for $H=-1 / 2$ (the phantom sector) and $N=1,0.9,0.8$. 


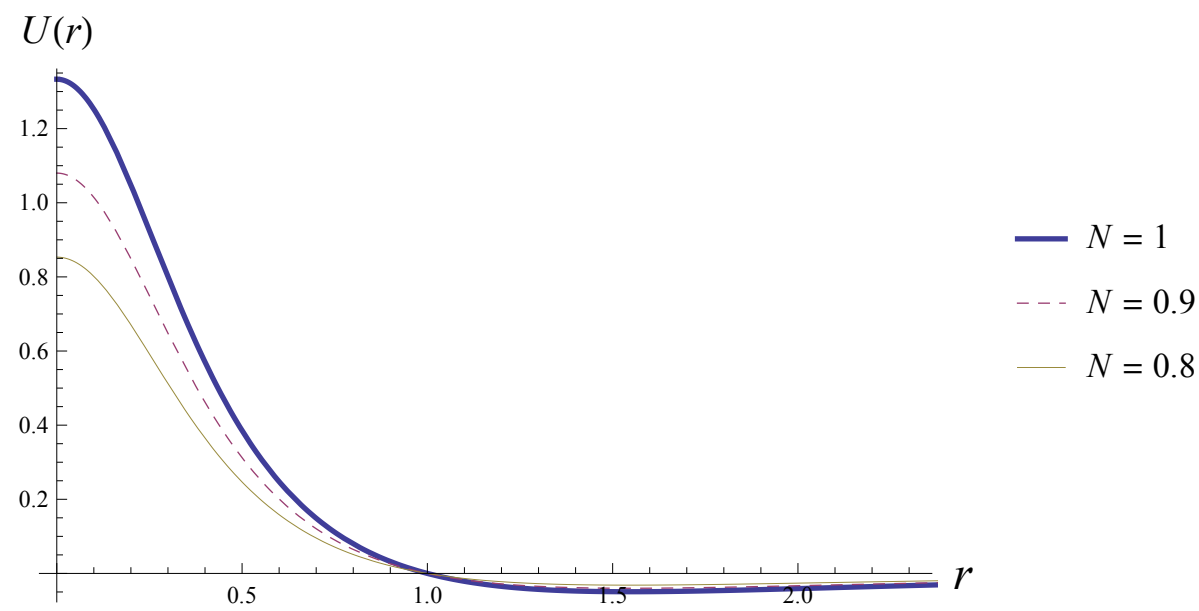

Figure 5. The potential $U(r)$ in the Einstein frame for $H=-1 / 2$ (phantom sector) and $N=1,0.9,0.8$.

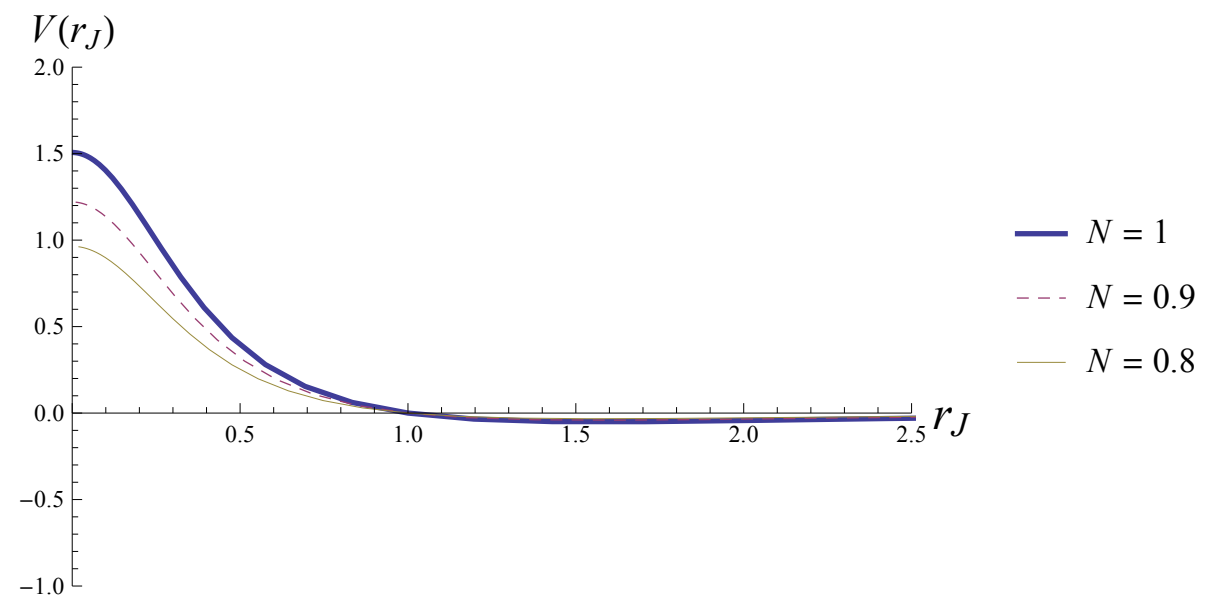

Figure 6. The potential $V\left(r_{\mathrm{J}}\right)$ in the Jordan frame for $H=-1 / 2$ (phantom sector) and $N=1,0.9,0.8$.

It is easy to show that if we slightly change the ansatz (62) by replacing $\cosh ^{2}(k \alpha)$ in the denominator with $\cosh ^{p}(k \alpha), p>2$, we will obtain stringlike models with potentials vanishing on the symmetry axis.

\section{Discussion}

We have considered the opportunity of obtaining stringlike, or solitonic vacuum solutions with cylindrical symmetry in the framework of hybrid metric-Palatini gravity (HMPG) without specifying the dependence $f(\mathcal{R})$ or, equivalently, the potential $V(\phi)$ in the STT representation of this theory. In addition to producing some particular examples of such configurations, we have obtained some results of more general significance:

1. Equations (45) and (51) implying that stringlike solutions cannot be obtained with purely nonpositive or purely nonnegative potentials.

2. Vacuum static cylindrically symmetric solutions with a regular asymptotic or a regular axis are necessarily boost-invariant in the $(z, t)$ subspace.

3. If the conformal factor $\mathrm{e}^{2 \eta}$ between $\mathbb{M}_{\mathrm{J}}$ and $\mathbb{M}_{\mathrm{E}}$ is regular in the whole range $x \in \mathbb{R}$ and finite in the limits $x \rightarrow \pm \infty$, then solitonic solutions simultaneously exist in $\mathbb{M}_{\mathrm{J}}$ and in $\mathbb{M}_{\mathrm{E}}$ and they are characterized by the same deficit angle. 
All of these observations are not restricted to the STT representation of HMPG but apply to an arbitrary STT from the class (5); moreover, they apply to arbitrary $f(R)$ theories of gravity, since the latter are known to coincide with the Brans-Dicke STT with the coupling constant $\omega=0$ and a nonzero scalar field potential whose form depends on the function $f(R)[4,5]$.

Here we have discussed only static cylindrically symmetric configurations, but probably of even greater interest can be stringlike models with rotation, for which numerous solutions are known in GR (see, e.g., $[23,24,44])$, and their extensions to HMPG are quite possible.

In addition to stringlike solitons with a regular axis, possible objects of interest, which can look from outside like cosmic strings, are cylindrical wormholes which are also globally regular configurations, but that do not contain a symmetry axis at all, similarly to spherically symmetric wormholes which do not contain a center of symmetry. Instead of an axis, where the circular radius $r$ turns to zero, cylindrical wormholes have a minimum of $r$ and, around it, two regions with much larger values of $r[45,46]$.

It is well known that the existence of wormholes in GR, in general, requires some amount of "exotic" matter violating the standard (Weak and Null) energy conditions, and its necessity can be avoided either in alternative theories of gravity (see $[47,48]$ for reviews) or within general relativity in cylindrical symmetry by invoking rotation and an appropriate choice of matter sources [49-51]. In this respect, we can note that HMPG, being a purely geometric source of scalar fields, both canonical and phantom, can provide a natural framework for wormhole construction without other "exotic" sources. Within spherical symmetry, this has already been confirmed [14,15], and a similar consideration in cylindrical symmetry can be one of the subjects of our further studies.

Author Contributions: Conceptualization and methodology, K.B.; investigation, K.B., S.B., M.S.; writing-original draft preparation, K.B., S.B.; writing—review and editing, K.B., M.S.; visualization, K.B., S.B., M.S. All authors have read and agreed to the published version of the manuscript.

Funding: The work was funded by the RUDN University Program 5-100 and the Russian Basic Research Foundation grant 19-02-0346. The work of K.B. was also partly performed within the framework of the Center FRPP supported by MEPhI Academic Excellence Project (contract No. 02.a03.21.0005, 27.08.2013).

Acknowledgments: The authors are grateful to the reviewers for their useful comments, critical remarks and advice.

Conflicts of Interest: The authors declare no conflict of interest.

\section{References}

1. Ishak, M. Testing general relativity in cosmology. Living Rev. Relativ. 2019, 22, 1. [CrossRef] [PubMed]

2. Basak, S.; et al. [Planck Collaboration]. Planck 2018 results. VI. Cosmological parameters. Astron. Astrophys. 2018, 641, 6 .

3. Copeland, E.J.; Sami, M.; Tsujikawa, S. Dynamics of dark energy. Int. J. Mod. Phys. D 2006, 15, 1753-1835. [CrossRef]

4. Capozziello, S.; De Laurentis, M. Extended Theories of Gravity. Phys. Rep. 2011, 509, 167-321. [CrossRef]

5. Nojiri, S.-I.; Odintsov, S.D. Introduction to modified gravity and gravitational alternative for dark energy. Int. J. Geom. Meth. Mod. Phys. 2007, 4, 115. [CrossRef]

6. Harko, T.; Koivisto, T.S.; Lobo, F.S.N.; Olmo, G.J. Metric-Palatini gravity unifying local constraints and late-time cosmic acceleration. Phys. Rev. D 2012, 85, 084016. [CrossRef]

7. Capozziello, S.; Harko, T.; Koivisto, T.S.; Lobo, F.S.N.; Olmo, G.J. Hybrid metric-Palatini gravity. Universe 2015, 1, 199. [CrossRef]

8. Harko, T.; Lobo, F.S.N. Extensions of $f(R)$ Gravity: Curvature-Matter Couplings and Hybrid Metric-Palatini Theory; Cambridge University Press: Cambridge, UK, 2018.

9. Harko, T.; Lobo, F.S.N. Beyond Einstein's General Relativity: Hybrid metric-Palatini gravity and curvature-matter couplings. Int. J. Mod. Phys. D 2020. Available online: https://arxiv.org/pdf/2007. 15345.pdf (accessed on 10 October 2020).

10. Capozziello, S.; Harko, T.; Lobo, F.S.N.; Olmo, G.J. Hybrid modified gravity unifying local tests, galactic dynamics and late-time cosmic acceleration. Int. J. Mod. Phys. D 2013, 22, 1342006. [CrossRef] 
11. Capozziello, S.; Harko, T.; Koivisto, T.S.; Lobo, F.S.N.; Olmo, G.J. Cosmology of hybrid metric-Palatini f(X)-gravity. J. Cosmol. Astropart. Phys. 2013, 2013, 011. [CrossRef]

12. Edery, A.; Nakayama, Y. Palatini formulation of pure $R^{2}$ gravity yields Einstein gravity with no massless scalar. Phys. Rev. D 2019, 99, 124018. [CrossRef]

13. Dǎnilă, B.; Harko, T.; Lobo, F.S.N.; Mak, M.K. Spherically symmetric static vacuum solutions in hybrid metric-Palatini gravity. Phys. Rev. D 2019, 99, 064028. [CrossRef]

14. Bronnikov, K.A. Spherically symmetric black holes and wormholes in hybrid metric-Palatini gravity. Grav. Cosmol. 2019, 25, 331. [CrossRef]

15. Bronnikov, K.A.; Bolokhov, S.V.; Skvortsova, M.V. Hybrid metric-Palatini gravity: black holes, wormholes, singularities and instabilities. Grav. Cosmol. 2020, 26, 212. [CrossRef]

16. Harko, T.; Lobo, F.S.N.; da Silva, H.M.R. Cosmic stringlike objects in hybrid metric-Palatini gravity. Phys. Rev. D 2020, 101, 124050. [CrossRef]

17. Böhmer, C.G.; Tamanini, N. Generalized hybrid metric-Palatini gravity. Phys. Rev. D 2013, 87, 084031.

18. Bombacigno, F.; Moretti, F.; Montani, G. Scalar modes in extended hybrid metric-Palatini gravity: Weak field phenomenology. Phys. Rev. D 2019, 100, 124036. [CrossRef]

19. Rosa, J.L.; Carloni, S.; Lemos, J.P.S. The cosmological phase space of generalized hybrid metric-Palatini theories of gravity. Phys. Rev. D 2020, 101, 104056. [CrossRef]

20. Rosa, J.L.; Lemos, J.P.S.; Lobo, F.S.N. Stability of Kerr black holes in generalized hybrid metric-Palatini gravity. Phys. Rev. D 2020, 101, 044055. [CrossRef]

21. Vilenkin, A. Gravitational field of vacuum domain walls and strings. Phys. Rev. D 1981, 23, 852. [CrossRef]

22. Bronnikov, K.A.; Shikin, G.N. Cylindrically symmetric solitons with nonlinear self-gravitating scalar fields. Grav. Cosmol. 2001, 6, 231.

23. Stephani, H.; Kramer, D.; MacCallum, M.A.H.; Hoenselaers, C.; Herlt, E. Exact Solutions of Einstein's Field Equations; Cambridge Monographs on Mathematical Physics; Cambridge University Press: Cambridge, UK, 2009.

24. Bronnikov, K.A.; Santos, N.O.; Wang, A. Cylindrical systems in general relativity. Class. Quantum Grav. 2020, 37, 113002. [CrossRef]

25. Bergmann, P.G. Comments on the scalar-tensor theory. Int. J. Theor. Phys. 1968, 1, 25. [CrossRef]

26. Wagoner, R. Scalar-tensor theory and gravitational waves. Phys. Rev. D 1970, 1, 3209. [CrossRef]

27. Nordtvedt, K. Post-Newtonian metric for a general class of scalar-tensor gravitational theories and observational consequences. Astrophys. J. 1970, 161, 1059. [CrossRef]

28. Penrose, R. Conformal treatment of the infinity. In Relativity, Groups and Topology; DeWitt, C., DeWitt, B., Eds.; Gordon and Breach: London, UK, 1964; p. 565.

29. Chernikov, N.A.; Tagirov, E.A. Quantum theory of scalar field in de Sitter space-time. Ann. Inst. H. Poincare Phys. Theor. A 1968, 9, 109.

30. Zaitsev, N.A.; Kolesnikov, S.M. Self-consistent interaction of scalar and tensor gravitational fields. In Problems of Gravitation Theory and Particle Theory, Issue 4; Staniukovich, K.P., Sokolik, G.A., Eds.; Atomizdat: Moscow, Russia, 1970; pp. 24-50. (In Russian)

31. Bronnikov, K.A. Scalar vacuum structure in general relativity and alternative theories. Conformal continuations. Acta Phys. Polon. B 2001, 32, 3571.

32. Bronnikov, K.A. Scalar-tensor gravity and conformal continuations. J. Math. Phys. 2002, 43, 6096. [CrossRef]

33. Bronnikov, K.A.; Chernakova, M.S. Generalized theory of gravity and conformal continuations. Grav. Cosmol. 2005, 11, 305-309.

34. Bocharova, N.M.; Bronnikov, K.A.; Melnikov, V.N. On an exact solution of the Einstein-scalar field equations. Vestn. Mosk Univ., Fiz. Astron. 1970, 6, 706.

35. Bronnikov, K.A. Scalar-tensor theory and scalar charge. Acta Phys. Pol. B 1973, 4, 251.

36. Bronnikov, K.A.; Clement, G.; Constantinidis, C.P.; Fabris, J.C. Structure and stability of cold scalar-tensor black holes. Phys. Lett. A 1998, 243, 121. [CrossRef]

37. Bronnikov, K.A.; Chernakova, M.S.; Fabris, J.C.; Pinto-Neto, N.; Rodrigues, M.E. Cold black holes and conformal continuations. Int. J. Mod. Phys. D 2008, 17, 25. [CrossRef]

38. Kamke, E. Differentialgleichungen: Lösungsmethoden und Lösungen. I. Gewönliche Differentialgleichungen; Springer International Publishing: Cham, Switzerland, 1979. 
39. Bronnikov, K.A. Static fluid cylinders and plane layers in general relativity. J. Phys. A Math. Gen. 1979, 12, 201. [CrossRef]

40. Bronnikov, K.A.; Sushkov, S.V. Trapped ghosts: A new class of wormholes. Class. Quantum Grav. 2010, 27, 095022. [CrossRef]

41. Bronnikov, K.A. Trapped ghosts as sources for wormholes and regular black holes. The stability problem. In Wormholes, Warp Drives and Energy Conditions; Lobo, F.S.N., Ed.; Springer: New York, NY, USA, 2017; pp. 137-159.

42. Bronnikov, K.A. Scalar fields as sources for wormholes and regular black holes. Particles 2018, 1, 56-81. [CrossRef]

43. Levi-Civita, T. $d s^{2}$ einsteiniani in campi newtoniani. IX: L'analogo del potenziale logaritmico. Rend. Accad. Lincei 1919, 28, 101-109.

44. Bolokhov, S.V.; Bronnikov, K.A.; Skvortsova, M.V. Rotating cylinders with anisotropic fluids in general relativity. Grav. Cosmol. 2019, 25, 122-130. [CrossRef]

45. Bronnikov, K.A.; Lemos, J.P.S. Cylindrical wormholes. Phys. Rev. D 2009, 79, 104089. [CrossRef]

46. Bronnikov, K.A.; Krechet, V.G.; Lemos, J.P.S. Rotating cylindrical wormholes. Phys. Rev. D 2013, 87, 084060. [CrossRef]

47. Visser, M. Lorentzian Wormholes: From Einstein to Hawking; AIP: Woodbury, NY, USA, 1995.

48. Lobo, F.S.N. (Ed.) Wormholes, Warp Drives and Energy Conditions; Springer: Berlin, Germany, 2017.

49. Bronnikov, K.A.; Krechet, V.G. Potentially observable cylindrical wormholes without exotic matter in GR. Phys. Rev. D 2019, 99, 084051. [CrossRef]

50. Bolokhov, S.V.; Bronnikov, K.A.; Skvortsova, M.V. Cylindrical wormholes: A search for viable phantom-free models in GR. Int. J. Mod. Phys. D 2019, 28, 1941008.

51. Bronnikov, K.A.; Krechet, V.G.; Oshurko, V.B. Rotating Melvin-like universes and wormholes in general relativity. Symmetry 2020, 12, 1306. [CrossRef]

(C) 2020 by the authors. Licensee MDPI, Basel, Switzerland. This article is an open access article distributed under the terms and conditions of the Creative Commons Attribution (CC BY) license (http://creativecommons.org/licenses/by/4.0/). 